\title{
A case report on phenobarbitone induced stevens-johnson syndrome: an alarming hypersensitivity reaction
}

\author{
Zankar D.R. ${ }^{1}$, Sheth H.J. ${ }^{2}$, Chaudhary R.G. ${ }^{3}$, Malhotra S.D. ${ }^{4}$, Patel P.R. ${ }^{5}$ \\ ${ }^{1}$ Dr. Dhara R. Zankat, $2^{\text {nd }}$ year Resident, Department of Dermatology, ${ }^{2}$ Dr. Haiya J. Sheth, $2^{\text {nd }}$ year Resident, Department \\ of Pharmacology, ${ }^{3}$ Dr. Raju G. Chaudhary, Professor \& Head, Department of Dermatology, ${ }^{4}$ Dr. Supriya D. Malhotra, \\ Professor \& Head, Department of Pharmacology, ${ }^{5}$ Dr. Pankaj R. Patel, Dean, Smt. NHL Municipal Medical College, \\ V.S. General Hospital, Ellisbridge, Ahmedabad Gujarat, India.
}

Corresponding Author: Dr. Haiya J. Sheth, $2^{\text {nd }}$ Year Resident, Department of Pharmacology, Smt. NHL Municipal Medical College, Ellisbridge, Ahmedabad, Gujarat, India. Email: haiyasheth@yahoo.com

\begin{abstract}
Stevens-Johnson syndrome (SJS) is an Ig E mediated hyper sensitivity reaction, sometimes complicated by ocular manifestations. Antiepileptics induced SJS is common with carbamazepine. Phenobarbitone is known to cause hypersensitivity reactions like mild to moderate rashes but not life-threatening reactions like SJS. We present a case report of an 18 years old female patient who presented with chief complaints of multiple, fluid-filled lesions associated with itching all over the body for 20 days and inability to open her eyes for 18 days. It had developed following ingestion of Tab. Phenobarbitone $30 \mathrm{mg}$ orally BD for 15 days. She was diagnosed as a case of SJS and treated with parenteral followed by oral corticosteroids and antihistaminics and recovered over a span of 20 days. Causality was assessed as per WHO-UMC Causality Assessment criteria and Naranjo Scale. Main pathogenesis is apoptosis through an interaction between cell-surface death receptor like Fas and its receptive ligand or due to genetic deficiency. In case of aromatic anticonvulsants, cross reactivity is also suspected. Genetic studies and cross reactivity testing can help prevent further incidences in few.
\end{abstract}

Key-words: Apoptosis, Causality Assessment, Corticosteroids, Phenobarbitone, Stevens-Johnson Syndrome

\section{Introduction}

Stevens-Johnson syndrome (SJS) is an Ig E mediated hyper sensitivity reaction characterized by erythematous macules or flat, atypical targetoid lesions with epidermal detachment of $<10 \%$ body surface area, often complicated by ocular conjunctivitis or uveitis and symblepharon formation [1].

Drug induced SJS is commonly encountered with antibacterials, antiepileptic drugs (AEDs), non-steroidal anti-inflammatory drugs and oxide inhibitors [2].

Here, drug suspected is Phenobarbitone. Owing to low toxicity and inexpensiveness, it is still one of the most widely prescribed AEDs [3]. With Phenobarbitone, sedation is most frequent Adverse Drug Reaction (ADR) [4]. Hyper sensitivity reactions like maculapapular, morbilliform, or scarlatiniform rashes with phenobarbitone have been reported in 1-3\% patients [4]. However, severe and life-threatening reactions like SJS and Toxic Epidermal Necrolysis (TEN) are extremely rare. Greatest risk for developing SJS is usually in first two months of commencing phenobarbitone [1].

Here, we present a case of Phenobarbitone, being the suspected drug for SJS in an adolescent female suffering from epilepsy.

\section{Case History}

An 18 years old female patient presented to the Skin Outpatient Department at our setup with chief complaints of multiple, few well-defined and fluid-filled lesions associated with itching all over the body since 20 days and inability to 


\section{Case Report}

open her eyes since 18 days. These fluid-filled lesions initially developed over her left hand gradually involving her right hand, face, chest, abdomen, back and lower limbs bilaterally. The lesions began to rupture on their own within few days, leaving behind raw and erythematous lesions all over the body. Ophthalmic examination showed oedema and erythema over and under the eyelids. Oral mucosa was also ulcerated. These lesions had developed following the ingestion of Tab. Phenobarbitone $30 \mathrm{mg}$ orally BD for 15 days.

On further eliciting the history, patient informed that she had been diagnosed with Epilepsy 1.5 years back and was on Tab. Valproate $200 \mathrm{mg}$ orally TDS since then. During that period, no history of hypersensitivity is recorded. Once the antiepileptic drug was changed from Tab. Valproate to Tab. Phenobarbitone, patient developed fluid-filled lesions 15 days after commencing Tab. Phenobarbitone. Thus the attending dermatologist diagnosed it as a case of Phenobarbitone induced Stevens Johnson Syndrome.

She was admitted immediately for further treatment and kept under observation. She was infused with Intravenous fluid (DNS), Inj. Avil (Pheniramine maleate), Inj. Dexona (Dexamethasone), Inj. Ceftriaxone, Inj. Rantac (Ranitidine). Fusicare cream was locally applied over the lips and erosions twice daily followed by Liquid Paraffin gauze over lips twice daily. She was also referred to Medicine Department where Tab. Phenobarbitone $30 \mathrm{mg}$ orally BD was withdrawn by the attending physician and changed her antiepileptic drug therapy to Tab. Levetiracetam $500 \mathrm{mg}$ orally BD.

With initiation of the treatment, gradually the skin lesions got crusted and eventually resulted in hyperpigmented erythematous plaques of varying sizes all over the body (see Figure 1). Inability to open her eyes during the initial days due to erythema and periorbital oedema also improved gradually with the treatment. The patient had recovered over a span of 20 days. At the time of discharge, she was also given a Drug List for future reference. This ADR was reported to the nearest ADR Monitoring Centre and uploaded via Vigiflow under the Pharmacovigilance Programme of India (PvPI) having report id: 2017-51921.

\section{Discussion}

Literature on SJS, a Type 1 Hypersensitivity reaction, was 1st jointly published in 1922 [5]. It's rare with an incidence of 0.05 - 2 persons per 1 million populations per year [2]. SJS, known as erythema multiforme major, represents a continuum of disease, most benign type of which is erythema multiforme, whereas toxic epidermal necrolysis is the most severe [2]. It has a potential risk for morbidity and mortality.

It can get precipitated by various factors such as infections (HIV, herpes); systemic diseases (collagen vascular disease); physical agents; foods and drugs. HLA-B12, HLA-B*5801, HLA-B*1502 are also involved with increased risk of developing SJS / TEN [2].

Among the AEDs responsible for inducing SJS, carbamazepine is the most commonly encountered AED. Antiepileptic hypersensitivity syndrome, comprising of fever, rash, and lymphadenopathy and less commonly lymphocytosis, and liver and other organ involvement, have been associated with some antiepileptics including phenobarbital [4]. However, reports on phenobarbitone being the culprit drug for SJS and TEN are relatively lesser. The relative risk for aromatic AEDs including phenobarbital to cause SJS is 11 to 15 [1]. This ratio indicates the probability of SJS in exposed/ non-exposed population. It is also noted that more than $90 \%$ of SJS and TEN cases have occurred in the first 63 days of AED use [6]. This is in context to our case where lesions had developed within two weeks of phenobarbitone ingestion.

The main pathogenesis for development of lesions is apoptosis. The keratinocytes undergo apoptosis through an interaction between cell-surface death receptor like Fas and its receptive ligand; induced by proinflammatory cytokines like TNF- $\alpha$, IL-6 and soluble CD40 ligand [1]. Drug hypersensitivity leads to major histocompatibility class I - restricted drug presentation and is followed by an expansion of cytotoxic $\mathrm{T}$ lymphocytes, leading to an infiltration of skin lesions with cytotoxic T-lymphocytes and natural killer cells. Granulysin probably is the key mediator for disseminated keratinocyte death in SJS/TEN [2].

Genetic deficiency or abnormality of epoxide hydroxylase enzyme may also lead to excessive accumulation of epoxides, thereby causing apoptosis. This is another suspected mechanism for above mentioned hypersensitivity reaction. These deficiencies damage cells by eliciting an immune response. Thus, genotyping studies can throw some light regarding the aetiology of SJS, whether drug induced or genetic. 


\section{Case Report}

Cross reactivity is also one possible mechanism. Studies have shown cross reactivity between Phenytoin and Carbamazepine. Main mechanism for cross-reactivity is thought to be due to accumulation of toxic hydroxylated aromatic metabolites [7]. Study by Mockenhaupt stated cross sensitivity between phenytoin \& phenobarbital is around $53.3 \%$ [6]. Thus, such patients should be shifted from aromatic to non-aromatic anticonvulsant therapy to prevent future incidences of hypersensitivity reactions.

Involvement of eyes and oral mucosa is a commonly encountered manifestation in SJS. Initially, lesions almost always involve mouth and lips which are usually ignored by the patients. If patients seek medical help at the initial stage itself, then SJS/TEN related morbid and mortal outcomes can be overcome. Conjunctivitis is reported in about $30 \%$ of children who develop SJS [5]. Ophthalmology consultation is a must or else the lesions may heal with scarring resulting in corneal vascularization or visual impairment. In this case, suspected drug, Phenobarbitone was withdrawn immediately. Thus, dechallenge was done. However, rechallenge was avoided. This is because, SJS itself is a life-threatening condition plus outcome post rechallenge would have proved fatal too. Also, it would have been unethical to perform rechallenge unless accidentally induced. Hence, causality was termed as probable/ likely according to WHO Uppsala Monitoring Centre causality assessment criteria because of lack of rechallenge information [8]. Naranjo causality assessment scale also showed a score of 7 , pointing towards probable causality [9].

Patient was kept under observation; managed well by I.V. fluids along with parenteral administration of corticosteroids and antihistaminics for one week. AED therapy was changed from Phenobarbitone to Levetiracetam by the physician. This is in reference to the study by Lee YJ which states that Levetiracetam is efficacious than Phenobarbitone in children with status epilepticus or acute repetitive seizure [10].

Looking at a speedy recovery of lesions, she was shifted to oral medications and discharged once all the lesions had crusted.

\section{Conclusion}

Mortality rates with SJS are relatively low compared to that with TEN. Thus, early recognition of manifestations of SJS followed by withdrawal of the causative drug and timely management can further reduce rates of morbidity and mortality in such patients. Patients need to be counselled regarding the severity and intensity of SJS as well as TEN and its outcome. Drug lists for preventing future episodes in the same patients are also a must. Aromatic anticonvulsants should be replaced by non-aromatic anticonvulsants in patients having suffered from hypersensitivity syndromes.

Cross reactivity testing and genotyping studies prior to starting AED therapy can also aid towards its prevention. HLA-B status of the patients can be determined by genotyping studies in-order to rule out genetic aetiology of SJS. However, such tests can be availed only at selective health care centres. Thus, it is ultimately in the hands of the prescriber to remain watchful for any adverse event.

Acknowledgement: We would like to thank Dr. S. T. Malhan Sir, the Medical Superintendent of our hospital for his constant support and guidance.

Contribution Details of Authors- Concept was given by Dr. Dhara Zankat, Dr. Haiya Sheth, Dr. Raju Chaudhary, Dr. Supriya Malhotra. Literature search and manuscript preparation by Dr. Dhara Zankat, Dr. Haiya Sheth. Editing and Review of manuscript by Dr. Raju Chaudhary, Dr. Supriya Malhotra and Dr. Pankaj Patel.

\begin{tabular}{|c|c|c|c|c|c|}
\hline & Contributor & Contributor & Contributor & Contributor & Contributor \\
& $\mathbf{1}$ & $\mathbf{2}$ & $\mathbf{3}$ & $\mathbf{4}$ & $\checkmark$ \\
\hline Concepts & $\checkmark$ & $\checkmark$ & $\checkmark$ & $\checkmark$ & \\
\hline Design & $\checkmark$ & $\checkmark$ & & $\checkmark$ & \\
\hline Definition of intellectual content & & & $\checkmark$ & & \\
\hline Literature search & $\checkmark$ & $\checkmark$ & & & \\
\hline Manuscript preparation & $\checkmark$ & $\checkmark$ & $\checkmark$ & $\checkmark$ & \\
\hline Manuscript editing & & & & & $\checkmark$ \\
\hline Manuscript review & & & & & $\checkmark$ \\
\hline Guarantor & & & & & \\
\hline
\end{tabular}




\section{Case Report}

Funding: Nil, Conflict of interest: None

Permission of IRB: Yes

\section{References}

1. Gaur S, Agnihotri R. Phenobarbital induced StevensJohnson syndrome in a child. Indian Journal of Pharmacology. 2012; 44 (4):531-532.

2. Deore SS, Dandekar RC, Mahajan AM, Shiledar VV. Drug Induced - Stevens Johnson Syndrome: A Case Report. Int J Sci Stud. 2014;2(4):84-87.

3. Ramineni HB, Eluri P, Vipparla K, Suryadevara V. Phenobarbital induced Stevens Johnson syndrome: a case report. Int J Res Med Sci. 2015; 3:492-3.

4. Sean C Sweet man. Martindale: The Complete Drug Reference. 36th ed. London: Pharmaceutical Press; 2009. Antiepileptics: 492-495.

5. Stevens-Johnson syndrome. (2017, October 25). In Wikipedia, The Free Encyclopedia. Retrieved 07:10, October 30, 2017, from https:// en. wikipedia. org/w/ index. php? title=Stevens $\%$ E2\% 80\% 93 Johnson_ syndrome \& oldid $=807068005$.
6. Mockenhaupt M, Messenheimer J, Tennis P, Schlingmann J. Risk of Stevens-Johnson syndrome and toxic epidermal necrolysis in newusers of antiepileptics. Neurology.2005Apr12;64(7):1134-8.

7. Trivedi BS, Darji NH, Malhotra SD, Patel PR. AntiepilepticDrugs-induced Stevens-Johnson syndrome : A case Series. Journal of Basic and Clinical Pharmacy. 2016;8(1):42-44.

8. WHO. The use of the WHO-UMC system for standardized case causality assessment, 2014. Available at: http://www.WHOUMC.org/graphics/4409.pdf

9. Naranjo CA, Busto U, Sellers EM, Sandor P, Ruiz I, Roberts EA, Janecek E, Domecq C, Greenblatt DJ. A method for estimating the probability of adverse drug reactions. Clin Pharmacol Ther. 1981 Aug; 30 (2): 239-45.

10. Lee Y-J, Yum M-S, Kim E-H, Ko T-S. Intravenous levetiracetam versus phenobarbital in children with status epilepticus or acute repetitive seizures. Korean Journal of Pediatrics. 2016; 59(1):35-39.

\section{How to cite this article?}

Zankar D.R, Sheth H.J, Chaudhary R.G, Malhotra S.D, Patel P.R. A case report on phenobarbitone induced stevensjohnson syndrome: an alarming hypersensitivity reaction. Int J Med Res Rev 2018;6 (03):200-203. doi:10.17511/ijmrr. 2018.103.12. 